\title{
Neutrino mass ordering obfuscated by the NSI
}

\author{
Francesco Capozzi, ${ }^{a}$ Sabya Sachi Chatterjee ${ }^{b, *}$ and Antonio Palazzo ${ }^{c, d}$ \\ ${ }^{a}$ Max-Planck-Institut für Physik (Werner-Heisenberg-Institut), Föhringer Ring 6, 80805 München, \\ Germany \\ ${ }^{b}$ Institute for Particle Physics Phenomenology, Department of Physics, Durham University, DH1 3LE, UK \\ ${ }^{c}$ Dipartimento Interateneo di Fisica "Michelangelo Merlin," Via Amendola 173, 70126 Bari, Italy \\ ${ }^{d}$ Istituto Nazionale di Fisica Nucleare, Sezione di Bari, Via Orabona 4, 70126 Bari, Italy \\ E-mail: capozzi@mpp.mpg.de, sabya.s.chatterjee@durham.ac.uk, \\ palazzo@ba.infn.it
}

The current data of the two long-baseline experiments $\mathrm{T} 2 \mathrm{~K}$ and $\mathrm{NO} v \mathrm{~A}$ indicate a $2.4 \sigma$ preference of normal neutrino mass ordering over the inverted mass ordering in case of standard $3 v$ analysis. However such a claim does not remain unaltered in presence of neutrino non-standard interactions (NSI) involving $e-\tau$ sector. We find that the sensitivity towards the neutrino mass ordering gets completely lost. So a rubust claim of the neutrino mass ordering requires a careful consideration of the impact of NSI.

40th International Conference on High Energy physics - ICHEP2020

July 28 - August 6, 2020

Prague, Czech Republic (virtual meeting)

\footnotetext{
${ }^{*}$ Speaker
} 


\section{Introduction}

Determination of the neutrino mass ordering (NMO) is one of the biggest priorities in the intensity frontier of high energy particle physics. To accomplish that goal a lot of efforts are being put together with the atmospheric, solar, reactor, and accelerator neutrinos. In the standard 3-flavor framework, NMO is defined to be normal if $m_{1}<m_{2}<m_{3}$, and inverted if $m_{3}<m_{1}<m_{2}$, where $m_{1}, m_{2}$, and $m_{3}$ are the masses of the three neutrino mass eigenstates $v_{1}, v_{2}$, and $v_{3}$ respectively. Interestingly, two long-baseline experiments $\mathrm{T} 2 \mathrm{~K}$ and $\mathrm{NO} v \mathrm{~A}$ are playing a leading role in this direction and provide $\mathrm{a} \sim 2.4 \sigma$ indication in favor of normal ordering $(\mathrm{NO})$ which we find in this work. In addition, we examine how the situation looks like in presence of non-standard interactions (NSI) of neutrinos with a special focus on the non-diagonal flavor changing type $\varepsilon_{e \tau}$ and $\varepsilon_{e \mu}$. We find that the present indication of $\mathrm{NO}$ in the standard 3-flavor framework gets completely vanished in the presence of NSI of the flavor changing type involving the $e-\tau$ flavors.

\section{Theoretical framework}

NSI may represent the low-energy manifestation of high-energy physics involving new heavy states (for a review see [1, 2]) or, alternatively, they can be mediated via the light mediators [3, 4]. As first recognized in [5], NSI of type neutral current (NC) can modify the dynamics [5, 6] of the neutrino flavor conversion in matter. It can be described by a dimension-six operator [5]

$$
\mathcal{L}_{\mathrm{NC}-\mathrm{NSI}}=-2 \sqrt{2} G_{F} \varepsilon_{\alpha \beta}^{f C}\left(\overline{\gamma_{\alpha}} \gamma^{\mu} P_{L} v_{\beta}\right)\left(\bar{f} \gamma_{\mu} P_{C} f\right),
$$

where $\alpha, \beta=e, \mu, \tau$ refer to the neutrino flavor, $f=e, u, d$ indicates the matter fermions, superscript $C=L, R$ denotes the chirality of the $f f$ current, and $\varepsilon_{\alpha \beta}^{f C}$ are the strengths of the NSI. The hermiticity of the interaction requires, $\varepsilon_{\beta \alpha}^{f C}=\left(\varepsilon_{\alpha \beta}^{f C}\right)^{*}$. For neutrino propagation in Earth matter, the relevant combinations are

$$
\varepsilon_{\alpha \beta} \equiv \sum_{f=e, u, d} \varepsilon_{\alpha \beta}^{f} \frac{N_{f}}{N_{e}} \equiv \sum_{f=e, u, d}\left(\varepsilon_{\alpha \beta}^{f L}+\varepsilon_{\alpha \beta}^{f R}\right) \frac{N_{f}}{N_{e}},
$$

where $N_{f}$ is the number density of fermion $f$. For the Earth, we can assume neutral and isoscalar matter, implying $N_{n} \simeq N_{p}=N_{e}$, in which case $N_{u} \simeq N_{d} \simeq 3 N_{e}$. Therefore, $\varepsilon_{\alpha \beta} \simeq \varepsilon_{\alpha \beta}^{e}+3 \varepsilon_{\alpha \beta}^{u}+$ $3 \varepsilon_{\alpha \beta}^{d}$. In presence of NSI, the effective Hamiltonian for neutrino propagation gets modified in matter, which in turn changes the probability of flavor conversion. For more details please see [7].

In this work, we only focus on the non-diagonal NSIs $\varepsilon_{e \mu}$ and $\varepsilon_{e \tau}$ along with their associated CP-phases $\phi_{e \mu}$ and $\phi_{e \tau}$ respectively. We recall that the current upper bounds (at 90\% C.L.) on the two NSI under consideration are: $\left|\varepsilon_{e \mu}\right| \lesssim 0.12$ and $\left|\varepsilon_{e \tau}\right| \lesssim 0.36$ as reported in the review [1], which refers to the global analysis [8]. These limits are basically corroborated by the more recent analysis [9] (for more details please refer to [7]). It is worth to mention that we do not focus on $\left|\epsilon_{\mu \tau}\right|$ as it has very strong upper bound $\left|\epsilon_{\mu \tau}\right|<8.0 \times 10^{-3}$ [10]. Let us now consider the appearance probability relevant for the two LBL experiments $\mathrm{T} 2 \mathrm{~K}$ and $\mathrm{NO} v \mathrm{~A}$ under consideration. In the presence of NSI, the appearance probability can be approximately written as a sum of three terms [11], $P_{\mu e} \simeq P_{0}+P_{1}+P_{2}$. The first two terms correspond to the standard 3-flavor probability while the third one arises due to NSI. Now considering the mixing angle $\theta_{13}$, the parameter $v\left(\equiv 2 V_{C C} E / \Delta m_{31}^{2}\right)$ and the coupling $|\varepsilon|$ are of the same order of magnitude $O(\epsilon)$ and small $(\sim 0.2)$ 
, while $\alpha \equiv \Delta m_{21}^{2} / \Delta m_{31}^{2}= \pm 0.03$ is $O\left(\epsilon^{2}\right)$, we can expand the above three terms as [12],

$$
\begin{aligned}
& P_{0} \simeq 4 s_{13}^{2} s_{23}^{2} f^{2} \\
& P_{1} \simeq 8 s_{13} s_{12} c_{12} s_{23} c_{23} \alpha f g \cos (\Delta+\delta) \\
& P_{2} \simeq 8 s_{13} s_{23} v|\varepsilon|\left[a f^{2} \cos (\delta+\phi)+b f g \cos (\Delta+\delta+\phi)\right],
\end{aligned}
$$

where $\Delta \equiv \Delta m_{31}^{2} L / 4 E$ is the atmospheric oscillating frequency, $V_{C C}=\sqrt{2} G_{F} N_{e}$ is the chargedcurrent matter potential, $L$ being the baseline and $E$ the neutrino energy. For compactness, we have used the notation $\left(s_{i j} \equiv \sin \theta_{i j}, c_{i j} \equiv \cos \theta_{i j}\right.$ ), and following [13], we have introduced

$$
\begin{aligned}
& f \equiv \frac{\sin [(1-v) \Delta]}{1-v}, \quad g \equiv \frac{\sin v \Delta}{v}, \\
& a=s_{23}^{2}, \quad b=c_{23}^{2}, \quad \text { if } \quad \varepsilon=\left|\varepsilon_{e \mu}\right| e^{i \phi_{e \mu}}, \\
& a=s_{23} c_{23}, \quad b=-s_{23} c_{23}, \quad \text { if } \quad \varepsilon=\left|\varepsilon_{e \tau}\right| e^{i \phi_{e \tau}} .
\end{aligned}
$$

In the expressions given in Eqs. (3)-(5) for $P_{0}, P_{1}$ and $P_{2}$, the sign of $\Delta, \alpha$ and $v$ is positive (negative) for NO (IO). For antineutrinos, the signs in front of the CP-phases and the matter parameter $v$ are flipped in Eqs. (3)-(5).
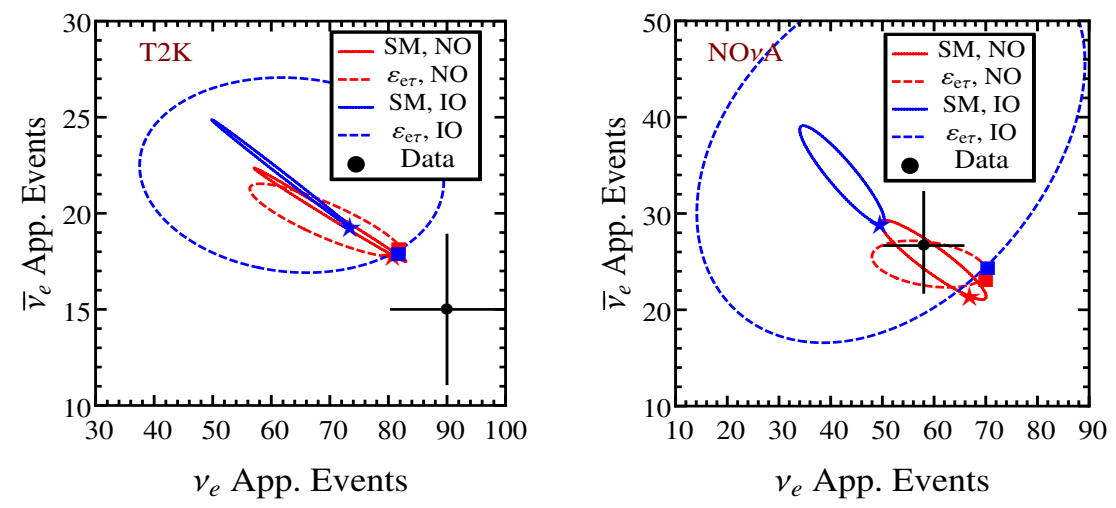

Figure 1: Bievents plot for the $\mathrm{T} 2 \mathrm{~K}$ (left panel) and $\mathrm{NO} v \mathrm{~A}$ (right panel). $\delta$ is the running parameter on each ellipse in the range $[0,2 \pi]$. The red (blue) star represents the best fit points for SM, NO (IO) determined by fitting the combination of the two experiments, and similarly the squares correspond to SM+NSI case. This figure has been taken from [7].

\section{Discussion at the level of the bievents plots}

Before we start the discussion, it is important to mention that for all the analyses presented here we have used the current data of $\mathrm{T} 2 \mathrm{~K}$ and $\mathrm{NO} v \mathrm{~A}$ extracted from [14] and [15]. In all our numerical simulations we have fixed the solar parameters $\theta_{12}$ and $\Delta m_{21}^{2}$ at the best fit point of the global analysis [16], while the reactor angle $\theta_{13}$ has been marginalized away with a strong $3.7 \% 1 \sigma$ prior on $\sin ^{2} \theta_{13}=0.0216$. For more details about the data sets and analysis procedures please see [7].

\footnotetext{
${ }^{1}$ Here we assume that the numerical analysis presented here points toward best fit values in the range $|\varepsilon| \sim 0.1-0.4$.
} 

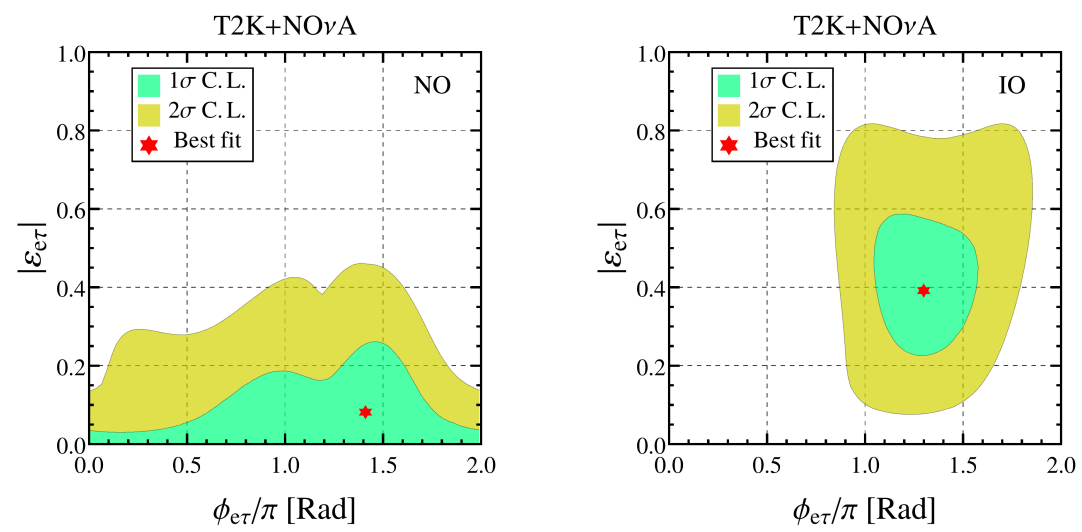

Figure 2: Allowed regions of the parameters $\left[\phi_{e \tau},\left|\varepsilon_{e \tau}\right|\right]$ determined by the combination of T2K and NOvA for NO (left panel) and IO (right panel). The contours are drawn at the $1 \sigma$ and $2 \sigma$ level for 1 d.o.f.. This figure has been taken from [7].

Fig. 1 represents the bievent plots for the two experiments $\mathrm{T} 2 \mathrm{~K}$ and $\mathrm{NO} v \mathrm{~A}$ in the context of the current data, where $x(y)$ axis corresponds to neutrino (antineutrino) appearance events. These plots are particularly very useful as they give a rough estimate of the situation of each experiment and clearly express the differences between the different experiments. Left (right) panel shows the bievent ellipses for T2K ( $\mathrm{NO} v \mathrm{~A})$. The continuous curves correspond to the SM framework and dashed curves correspond to the presence of SM+NSI, particularly $\varepsilon_{e \tau}$. SM (SM + NSI) ellipses have been generated with the fixed (those not marginalized) and the common best fit values of the oscillation parameters obtained from the combined analysis of the two experiments in SM (SM + NSI) framework where the standard CP-phase $\delta$ is the running parameter in the range $[0,2 \pi]$. The best fit values obtained from this analysis can be easily read from Fig. 3. The best fit value for $\Delta m_{31}^{2}$ (which is not shown in Fig. 3) obtained here in case SM is $2.5 \times 10^{-3}\left(2.46 \times 10^{-3}\right) \mathrm{eV}^{2}$ for NO (IO). Similarly in case of SM + NSI $\left(\varepsilon_{e \tau}\right)$ it is $2.49 \times 10^{-3}\left(2.46 \times 10^{-3}\right) \mathrm{eV}^{2}$ for NO (IO). The black dot points indicate the experimentally observed data for each experiment. Let us first comment about the SM results. One can see that in case of T2K, the NO best fit (red star) is closer to the data point with respect to the IO best fit (blue star), whereas in $\mathrm{NO} v \mathrm{~A}$ there is basically no particular preference of any of the two mass orderings. As a result, an overall global preference of NO is obtained at $2.4 \sigma$ as can be seen in Fig. 3. Now in the presence of NSI, the NO and IO best fit points (red and blue squares) for each experiment basically lie at the same distance from the experimental data points in contrast to the SM case. We find $\chi_{\mathrm{SM}+\mathrm{NSI} \text {, NO }}^{2}-\chi_{\mathrm{SM}+\mathrm{NSI}, \mathrm{IO}}^{2} \simeq 0.5$, corresponding to $0.7 \sigma$ preference of IO over NO (see also Fig. 3). Therefore, the indication in favor of NO found in the standard case gets completely lost in the presence of NSI. For more details please refer to [7].

\section{Numerical Results}

Fig. 2 shows the allowed regions determined by the combined analysis of $\mathrm{T} 2 \mathrm{~K}$ and $\mathrm{NO} v \mathrm{~A}$, in the plane spanned by $\left|\epsilon_{e \tau}\right|$ and $\phi_{e \tau}$. Left (right) panel corresponds to NO (IO). The red star in each panel denotes the best fit value. We have marginalized away the CP-phase $\delta$, the mixing angles $\theta_{13}$ (with prior) and $\theta_{23}$, and the squared-mass $\Delta m_{31}^{2}$. It is evident from the left panel that in case of NO, there is only $0.7 \sigma$ level preference of non-zero value of $\left|\epsilon_{e \tau}\right|$ with best fit value approximately 0.09 . Now in case of IO, we can see from the right panel there is a $2.5 \sigma$ level strong preference 

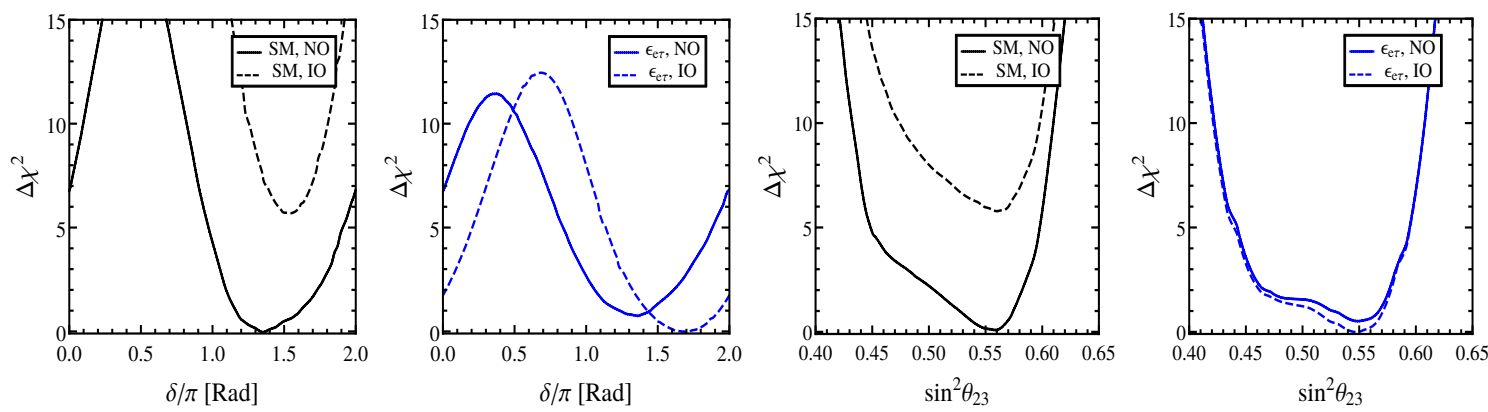

Figure 3: One dimensional $\Delta \chi^{2}$ projections of $\delta$ and $\theta_{23}$ for SM as well as SM+NSI framework, determined by the combination of $\mathrm{T} 2 \mathrm{~K}$ and $\mathrm{NO} v \mathrm{~A}$. This figure has been taken from [7].

of non-zero value of $\left|\epsilon_{e \tau}\right|$ with best fit value approximately 0.39 . The associated CP-phase $\phi_{e \tau}$ has the best fit value $1.42 \pi(1.30 \pi)$ for $\mathrm{NO}(\mathrm{IO})$.

In Fig. 3, we show the one-dimentional projections on two oscillation parameters $\delta$ and $\theta_{23}$ in the SM and SM+NSI framework. The allowed regions shown here are determined by the combined analysis of $\mathrm{T} 2 \mathrm{~K}$ and $\mathrm{NO} v \mathrm{~A}$. Solid (dashed) lines correspond to $\mathrm{NO}$ (IO). The undisplayed parameters in each plot have been marginalized away. One can observe that in the SM case there is a preference for $\mathrm{NO}$ at the $\sim 2.4 \sigma$ level. However this preference gets completely washed out and there is no preference of any ordering in the presence of NSI. It is worth to mention that the reconstruction of $\delta$ deteriorates in the presence of NSI and similarly the preferences for a non-maximal mixing and that for the higher octant of $\theta_{23}$ with respect to the lower octant found in the SM sensibly decrease, which can be attributed to the fact that in presence of NSI the number of degrees of freedom is higher than the SM case. Let us now make some comments regarding the NSI involving the $e-\mu$ sector. We have found that in this case NO is preferred over IO at the $2.5 \sigma$ level similar to the SM case. Therefore, the ambiguity of the indication in favor of the NO appears only when we consider the non-standard interactions in the $e-\tau$ sector. For more details please see [7].

\section{Conclusions}

In this work we have expounded the current data of $\mathrm{T} 2 \mathrm{~K}$ and $\mathrm{NO} v \mathrm{~A}$ in the presence of NSI involving the $e-\tau\left(\varepsilon_{e \tau}\right)$ and $e-\mu\left(\varepsilon_{e \mu}\right)$ sectors respectively. We found that the indication in favor of NO in SM gets completely vanished when one turns on the NSI with coupling strength $\left(\varepsilon_{e \tau}\right)$. It would be interesting to make the same analysis combining $\mathrm{NO} v \mathrm{~A}$ and $\mathrm{T} 2 \mathrm{~K}$ data with that of the existing atmospheric neutrino data. We also hope that the future high-statistics LBL experiments like DUNE and the atmospheric neutrino facilities might help to resolve this issue.

\section{Acknowledgments}

S.S.C. would like to thank the organizers of "40th International Conference on High Energy physics - ICHEP2020" for giving an opportunity to present this work. 


\section{References}

[1] Y. Farzan and M. Tortola, Neutrino oscillations and Non-Standard Interactions, Front.in Phys. 6 (2018) 10, [arXiv: 1710 . 09360].

[2] P. S. Bhupal Dev et al., Neutrino Non-Standard Interactions: A Status Report, in NTN Workshop on Neutrino Non-Standard Interactions St Louis, MO, USA, May 29-31, 2019, 2019. arXiv: 1907.00991.

[3] Y. Farzan, A model for large non-standard interactions of neutrinos leading to the LMA-Dark solution, Phys. Lett. B748 (2015) 311-315, [arXiv : 1505 . 06906].

[4] Y. Farzan and I. M. Shoemaker, Lepton Flavor Violating Non-Standard Interactions via Light Mediators, JHEP 07 (2016) 033, [arXiv: 1512 .09147].

[5] L. Wolfenstein, Neutrino Oscillations in Matter, Phys.Rev. D17 (1978) 2369-2374.

[6] S. Mikheev and A. Y. Smirnov, Resonance Amplification of Oscillations in Matter and Spectroscopy of Solar Neutrinos, Sov.J.Nucl.Phys. 42 (1985) 913-917.

[7] F. Capozzi, S. S. Chatterjee, and A. Palazzo, Neutrino Mass Ordering Obscured by Nonstandard Interactions, Phys. Rev. Lett. 124 (2020), no. 11 111801, [arXiv: 1908.06992].

[8] M. C. Gonzalez-Garcia and M. Maltoni, Determination of matter potential from global analysis of neutrino oscillation data, JHEP 09 (2013) 152, [arXiv: 1307 . 3092].

[9] I. Esteban, M. C. Gonzalez-Garcia, and M. Maltoni, On the Determination of Leptonic CP Violation and Neutrino Mass Ordering in Presence of Non-Standard Interactions: Present Status, JHEP 06 (2019) 055, [arXiv: 1905. 05203].

[10] IceCube Collaboration, M. G. Aartsen et al., Search for Nonstandard Neutrino Interactions with IceCube DeepCore, Phys. Rev. D97 (2018), no. 7 072009, [arXiv: 1709. 07079].

[11] T. Kikuchi, H. Minakata, and S. Uchinami, Perturbation Theory of Neutrino Oscillation with Nonstandard Neutrino Interactions, JHEP 0903 (2009) 114, [arXiv : 0809. 3312].

[12] J. Liao, D. Marfatia, and K. Whisnant, Degeneracies in long-baseline neutrino experiments from nonstandard interactions, Phys. Rev. D93 (2016), no. 9 093016, [arXiv: 1601.00927].

[13] V. Barger, D. Marfatia, and K. Whisnant, Breaking eight fold degeneracies in neutrino CP violation, mixing, and mass hierarchy, Phys. Rev. D65 (2002) 073023, [hep-ph/0112119].

[14] G. Żarnecki, Most Recent T2K Results on CPV in Neutrino Sector, Talk at Les Rencontres de Physique de la Vallée d'Aoste, 10-16 March 2019, La Thuile, Italy (2019).

[15] NOvA Collaboration, M. A. Acero et al., First measurement of neutrino oscillation parameters using neutrinos and antineutrinos by NOvA, arXiv: 1906.04907.

[16] F. Capozzi, E. Lisi, A. Marrone, and A. Palazzo, Current unknowns in the three neutrino framework, Prog. Part. Nucl. Phys. 102 (2018) 48-72, [arXiv : 1804 . 09678]. 